\title{
The ATLAS hadronic tau trigger
}

\author{
Curtis Black* \\ On behalf of the ATLAS Collaboration \\ University of Sydney \\ E-mail: c.black@physics.usyd.edu.au
}

\begin{abstract}
With the high luminosities of proton-proton collisions achieved at the LHC, the strategies for triggering have become more important than ever for physics analysis. The naive inclusive single tau lepton triggers now suffer from severe rate limitations. To allow for a large program of physics analyses with taus, the development of topological triggers that combine tau signatures with other measured quantities in the event is required. These combined triggers open many opportunities to study new physics beyond the Standard Model and to search for the Standard Model Higgs. We present the status and performance of the hadronic tau trigger in ATLAS. We demonstrate that the ATLAS tau trigger ran remarkably well over 2011, and how the lessons learned from 2011 led to numerous improvements in the preparation of the 2012 run. These improvements include the introduction of tau selection criteria that are robust against varying pileup scenarios, and the implementation of multivariate selection techniques in the tau trigger. First results from the improved 2012 tau trigger will be shown. Measurements of the trigger efficiency using the $Z \rightarrow \tau \tau$ and $W \rightarrow \tau \nu$ events are also presented.
\end{abstract}

36th International Conference on High Energy Physics

4-11 July 2012

Melbourne, Australia

${ }^{*}$ Speaker. 


\section{Introduction}

Tau leptons play an important role in the measurement of Standard Model (SM) physics and searches for Beyond the Standard Model (BSM) physics. Triggering on hadronic tau decays is therefore an essential ingredient of these measurements at the ATLAS Experiment [1] at the Large Hadron Collider (LHC). Tau triggers have been developed to identify events containing hadronically decaying tau leptons (denoted $\tau_{h}$ ), which enables analyses to use data enriched with tau candidates. Triggers have been developed to select events containing taus and other objects such as electrons, muons (which could originate from leptonic tau decays, denoted $\tau_{e / \mu}$ ) and missing transverse energy $\left(E_{T}^{\text {miss }}\right)$. Examples of results using tau triggers are shown in table 1.

\begin{tabular}{c|c|c} 
Trigger & Channel & Analysis \\
\hline \hline$\tau$ & $Z^{\prime} \rightarrow \tau_{h} \tau_{h}$ & High mass resonance search [2] \\
\hline$\tau+\tau$ & $H \rightarrow \tau_{h} \tau_{h}$ & Higgs search [3] \\
\hline$\tau+\mathrm{e} / \mu$ & $H \rightarrow \tau_{h} \tau_{e / \mu}$ & Higgs search [3] \\
& $Z \rightarrow \tau_{h} \tau_{e / \mu}$ & $Z$ cross section measurement [4] \\
\hline \multirow{2}{*}{$\tau+E_{T}^{\text {miss }}$} & $H^{+} \rightarrow \tau_{h} \nu$ & charged Higgs search [5] \\
& $W^{+} \rightarrow \tau_{h} v$ & $\tau$ polarisation measurement [6]
\end{tabular}

Table 1: Examples of analyses using hadronic tau triggers.

\section{ATLAS Trigger Design}

The ATLAS trigger [7] identifies interesting events along a chain of three levels of triggers, each applying an increasing level of data analysis complexity and sophistication. These layers are: the Level 1 hardware based trigger and the software based High Level Trigger (HLT) which consists of both the Level 2 and Event Filter triggers. A schematic of the ATLAS trigger structure is shown in figure 1(a). The trigger system is designed to reduce the input event rate of $40 \mathrm{MHz}$ to an output rate of $400 \mathrm{~Hz}$ capable of being written to storage without losing interesting physics events. This becomes increasingly challenging with the increasing luminosity delivered by the LHC as can be seen in figure 1(b) which shows the trigger rates for various trigger items as a function of instantaneous luminosity.

\subsection{Level 1}

The Level 1 trigger is a hardware based system using information provided by electromagnetic (EM) and hadronic (HAD) calorimeter towers with a spatial granularity of $\Delta \eta \times \Delta \phi=0.1 \times 0.1$, as shown in figure $1(\mathrm{c})$. Hadronically decaying taus are identified at L1 by the following features:

- Sum of energy in $2 \times 1$ pairs of EM towers

- Energy in $2 \times 2$ HAD towers behind the EM cluster

- Energy in a $4 \times 4$ isolation ring around the $2 \times 2$ core

For a given $\mathrm{L} 1$ item, a threshold is applied on the transverse energy, $E_{T}$, in the $2 \times 2$ core, for example, the L1_TAU11 item requires $E_{T}>11 \mathrm{GeV}$. To handle the large input rate, an isolation 


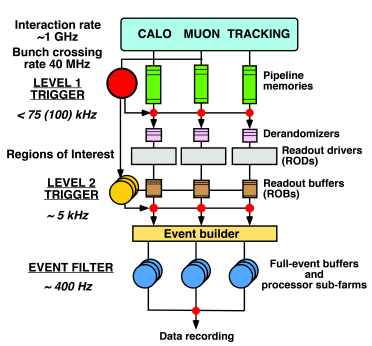

(a)

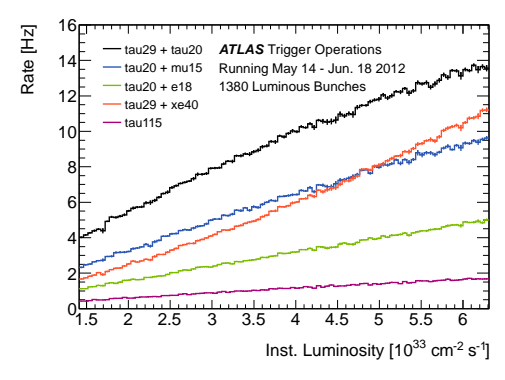

(b)

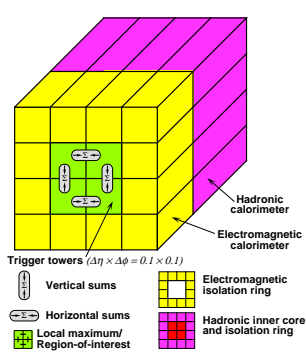

(c)

Figure 1: Block diagram of the data flow in the ATLAS trigger system (a), trigger rates as a function of instantaneous luminosity for tau triggers in 2012 (b) [8] and a schematic of the trigger towers used at Level 1 utilising both the EM and Hadronic calorimeters with a spatial granularity of $\Delta \eta \times \Delta \phi=0.1 \times 0.1$ (c).

criteria has to be applied. The position of the L1 deposit defines the Region of Interest (RoI) which is used in the HLT.

\subsection{Level 2}

At the Level 2 trigger, track reconstruction starts and is used along with the full detector granularity within the RoI. The L2 discriminates based on several shape variables measuring, for example, the energy weighted radius (figure 2(a)) of the tau decay products and the number of reconstructed tracks (figure 2(b)). The events shown in figure 2 were selected using a tag and probe selection to the $Z \rightarrow \tau \tau \rightarrow \mu \tau_{h}$ final state events as described by the tau identification efficiency measurement in [9].

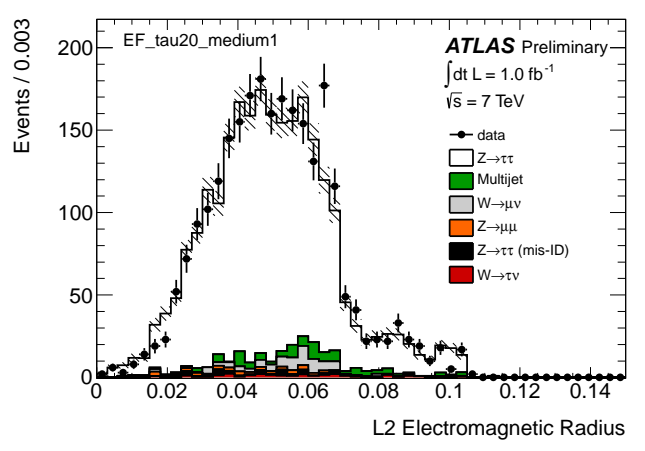

(a)

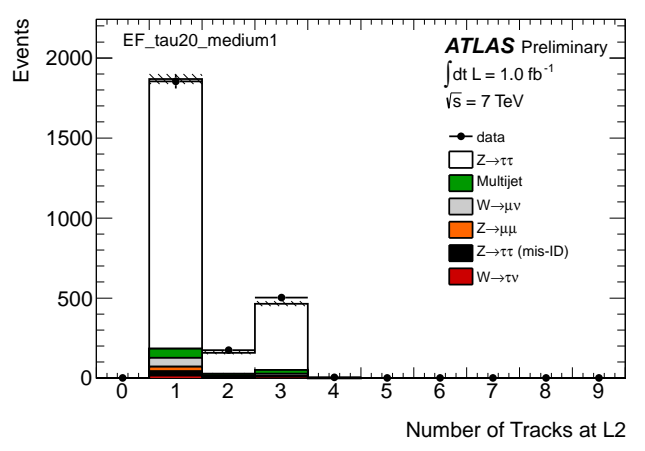

(b)

Figure 2: Tau decay shape variables calculated at Level 2 [8].

\subsection{Event Filter}

The third trigger level uses the complete event information to perform a full reconstruction of tau candidates using algorithms similar to those used in offline reconstruction. Figure 3 shows the comparison between the energy weighted radius calculated at the Event Filter and offline for signal Monte Carlo and background dijet data. The events selected here are the combined contributions from $Z \rightarrow \tau \tau, W \rightarrow \tau \nu$ and $Z^{\prime} \rightarrow \tau \tau$ signal Monte Carlo, the points represent data selected using a 
dijet selection criteria. The shift in the distribution is due to a less precise energy calibration at the Event Filter with respect to offline reconstruction.

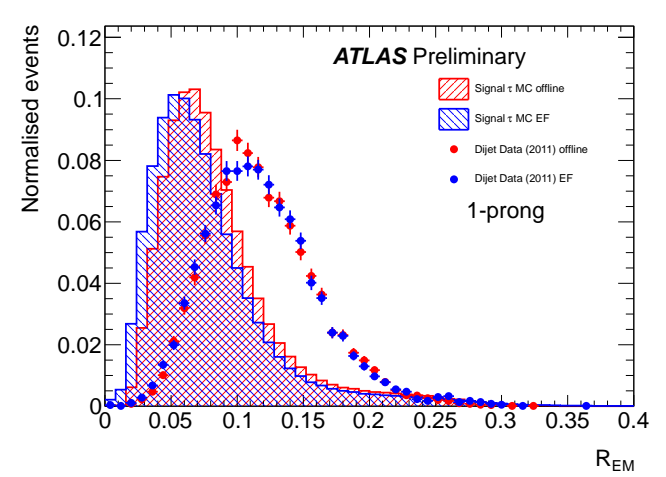

Figure 3: Distribution of radius of energy deposits in electromagnetic calorimeters, $R_{\mathrm{EM}}$, for Event Filter (EF) and offline reconstructed tau candidates with exactly one associated track (1prong) [8].

\section{Multivariate Based Tau Triggers}

In the 2012 data taking period, the event filter trigger utilizes two multivariate tau identification algorithms: the Boosted Decision Tree (BDT) and Log Likelihood (LLH) algorithms, similar to what is used in offline reconstruction. The performance of the new multivariate triggers with respect to offline identified tau candidates is shown for 1 prong (figure 4(a)) and 3 prong (figure 4(b)) tau candidates. The BDT and LLH decisions are optimised to achieve an efficiency of $85 \%$ and $80 \%$ for 1 and 3 prong tau candidates respectively.

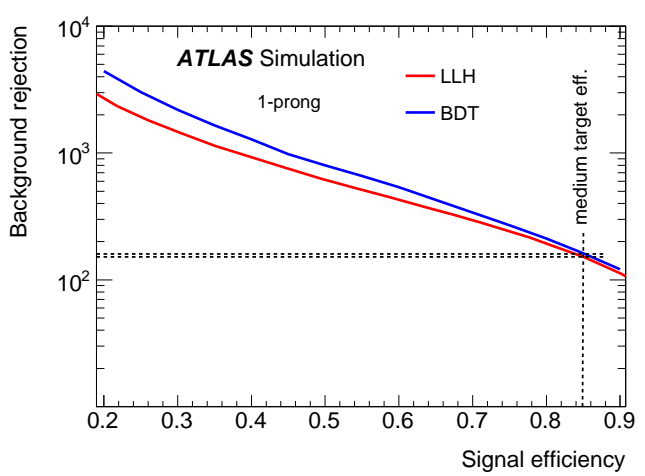

(a)

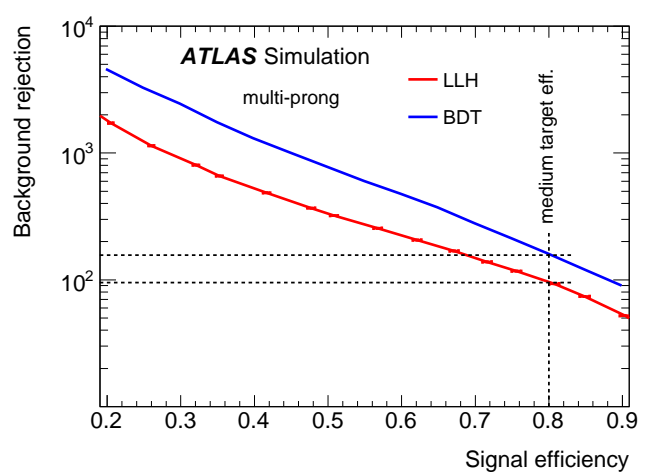

(b)

Figure 4: Signal efficiency vs background rejection rate for 1 (a) and 3 (b) prong tau candidates at the Event Filter using the new multivariate based discrimination methods [8].

\section{Tau Trigger Under High Pileup}

Tau trigger studies in 2011 showed a degradation of efficiency with increasing pileup. Figure 5(a) shows the efficiency degradation of the tau20_medium trigger in 2011 data. For the 2012 data taking period, the tau selection criteria were modified to provide robustness against the increasing pileup conditions. Figure 5(b) shows the efficiency with the new tau selection criteria in 2012 data. 


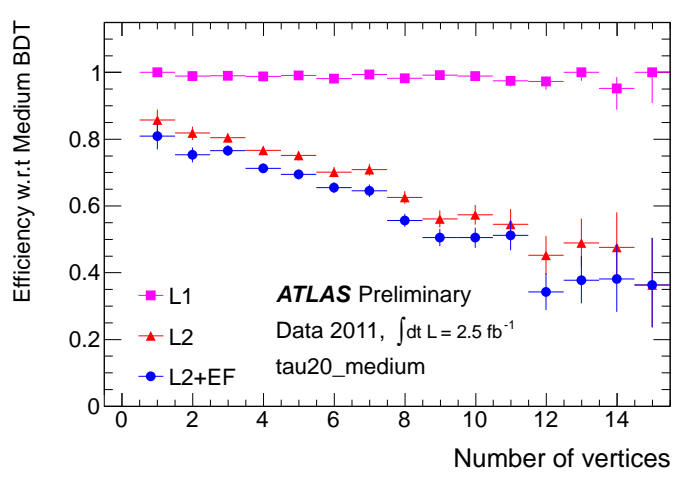

(a)

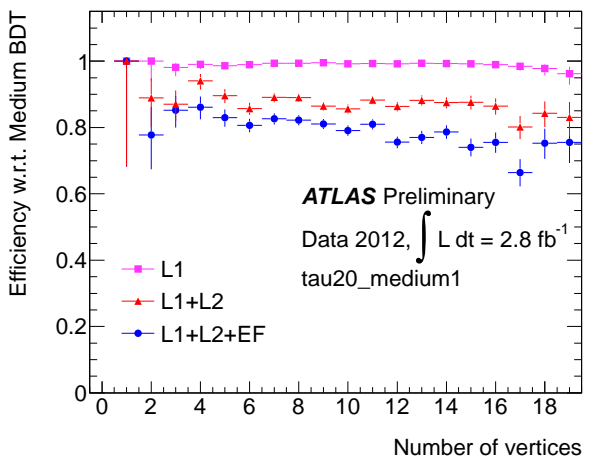

(b)

Figure 5: Performance of the tau20_medium trigger before (a) and after (b) the implementation of the new tau selection criteria [8].

The calorimeter cone size was decreased from 0.4 (in 2011) to 0.2 (in 2012), figure 6(a) shows an increase in marginal efficiency for the cut on the Level $2 \mathrm{EM}$ radius. Marginal efficiency, in this case, is the efficiency with respect to Level 1 for the cut on the Level 2 EM radius. A $\Delta Z$ selection, with respect to the leading track, was implemented in 2012 requiring $|\Delta Z|<2 \mathrm{~mm}$. Figure 6(b) shows the $\Delta Z$ distribution for signal Monte Carlo with an average of eight interactions per bunch crossing. The wide Gaussian distribution corresponds to pileup tracks while the central peak (displayed in the upper right corner) corresponds to the main interaction tracks.

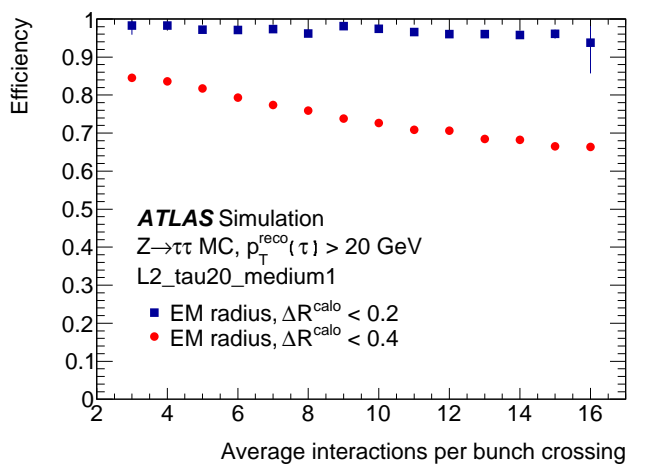

(a)

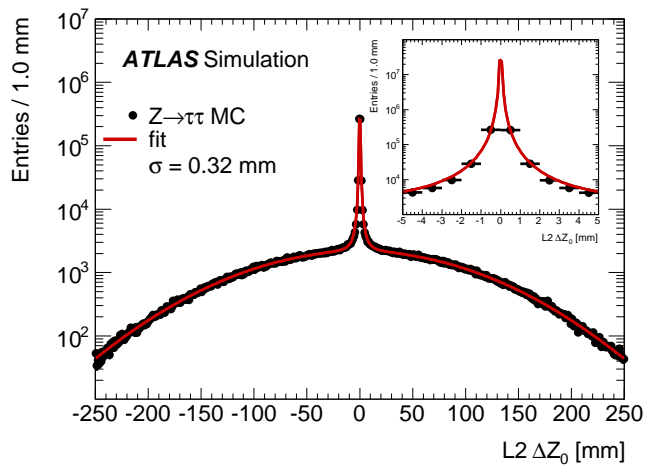

(b)

Figure 6: Marginal efficiencies for L2 EM radius calculated in cones of $\Delta R<0.2$ and 0.4 are shown as a function of the average number of interactions per bunch crossing (a) and the $\Delta Z$ distribution from $Z \rightarrow \tau \tau$ MC events with an average of eight interactions per bunch crossing (b) [8].

\section{Efficiency Measurements}

The performance of the tau trigger has been measured in 2012 data using a $Z \rightarrow \tau \tau \rightarrow \mu \tau_{h}$ tag and probe method. The efficiency is plotted in figure 7 with respect to the $p_{T}$ of offline tau candidates identified by the BDT algorithm. The term tau20_medium 1 implies a $20 \mathrm{GeV}$ requirement on the transverse energy, number of tracks restricted up to 3 and and medium selections on the BDT score at the event filter. 


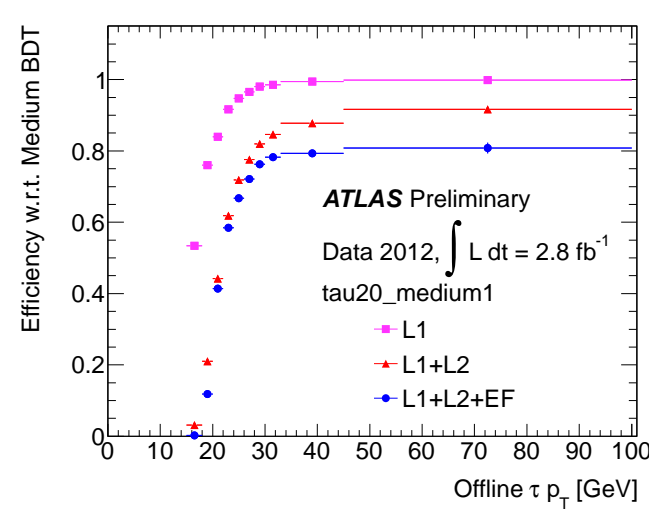

\section{Conclusions}

The ATLAS hadronic tau trigger successfully collected data in 2011. Many lessons were learned from 2011 which led to numerous improvements, including the introduction of tau selection criteria that are robust against pileup and the implementation of multivariate selection techniques in the tau trigger. These changes have proved to be successful in 2012.

\section{References}

[1] ATLAS Collaboration, "The ATLAS Experiment at the CERN Large Hadron Collider," 2008 JINST 3 S08003.

[2] ATLAS Collaboration, "A search for high-mass resonances decaying to $\tau^{+} \tau^{-}$in $p p$ collisions at $\sqrt{s}=7 \mathrm{TeV}$ with the ATLAS detector," 2012. CERN-PH-EP-2012-223

https://cdsweb.cern.ch/record/1489649/.

[3] ATLAS Collaboration, "Search for the Standard Model Higgs boson in the $H \rightarrow \tau^{+} \tau^{-}$decay mode with $4.7 \mathrm{fb}^{-1}$ of ATLAS data at $7 \mathrm{TeV}$," Mar 2012. ATLAS-CONF-2012-014 http://cdsweb.cern.ch/record/1429662/.

[4] ATLAS Collaboration, " $Z \rightarrow \tau \tau$ cross section measurement in proton-proton collisions at $7 \mathrm{TeV}$ with the ATLAS experiment," Feb 2012. ATLAS-CONF-2012-006 http://cdsweb.cern.ch/record/1426991/.

[5] ATLAS Collaboration, "Search for charged Higgs bosons decaying via $H^{+} \rightarrow \tau v$ in ttbar events using $4.6 \mathrm{fb}^{-1}$ of pp collision data at $\sqrt{s}=7 \mathrm{TeV}$ with the ATLAS detector," Mar 2012.

ATLAS-CONF-2012-011 http://cdsweb.cern.ch/record/1429659/.

[6] ATLAS Collaboration, "Measurement of Tau Polarization in $W \rightarrow \tau v$ Decays with the ATLAS Detector in pp Collisions at $\sqrt{s}=7 \mathrm{TeV}$," Mar 2012. ATLAS-CONF-2012-009 http://cdsweb.cern.ch/record/1428549/.

[7] ATLAS Collaboration, "Performance of the ATLAS Trigger System in 2010," Eur.Phys.J., vol. C72, p. 1849, 2012. hep-ex/1110.1530.

[8] ATLAS Collaboration, "Public Tau Trigger Results," http://twiki.cern.ch/twiki/bin/view/AtlasPublic/TauTriggerPublicResults/.

[9] ATLAS Collaboration, "Performance of the Reconstruction and Identification of Hadronic Tau Decays with ATLAS," Nov 2011. ATLAS-CONF-2011-152 http://cdsweb.cern.ch/record/1398195/. 